\title{
The energy impact of adaptive cruise control in real-world highway multiple-car- following scenarios
}

Yinglong $\mathrm{He}^{1}$, Michail Makridis² ${ }^{*}$, Georgios Fontaras ${ }^{2}$, Konstantinos Mattas ${ }^{2}$, Hongming $\mathrm{Xu}^{1}$ and Biagio Ciuffo ${ }^{2}$

\begin{abstract}
Background: Surging acceptance of adaptive cruise control (ACC) across the globe is further escalating concerns over its energy impact. Two questions have directed much of this project: how to distinguish ACC driving behaviour from that of the human driver and how to identify the ACC energy impact. As opposed to simulations or test-track experiments as described in previous studies, this work is unique because it was performed in real-world car-following scenarios with a variety of vehicle specifications, propulsion systems, drivers, and road and traffic conditions.
\end{abstract}

Methods: Tractive energy consumption serves as the energy impact indicator, ruling out the effect of the propulsion system. To further isolate the driving behaviour as the only possible contributor to tractive energy differences, two techniques are offered to normalize heterogeneous vehicle specifications and road and traffic conditions. Finally, ACC driving behaviour is compared with that of the human driver from transient and statistical perspectives. Its impact on tractive energy consumption is then evaluated from individual and platoon perspectives.

Results: Our data suggest that unlike human drivers, ACC followers lead to string instability. Their inability to absorb the speed overshoots may partly be explained by their high responsiveness from a control theory perspective. Statistical results might imply the followers in the automated or mixed traffic flow generally perform worse in reproducing the driving style of the preceding vehicle. On the individual level, ACC followers have tractive energy consumption $2.7-20.5 \%$ higher than those of human counterparts. On the platoon level, the tractive energy values of ACC followers tend to consecutively increase (11.2-17.3\%).

Conclusions: In general, therefore, ACC impacts negatively on tractive energy efficiency. This research provides a feasible path for evaluating the energy impact of ACC in real-world applications. Moreover, the findings have significant implications for ACC safety design when handling the stability-responsiveness trade-off.

Keywords: Tractive energy consumption, Adaptive cruise control, Multiple-car-following, Driving behaviour, Real-world data, String instability

\section{Introduction}

The energy, environmental and safety challenges facing humanity are further exacerbated by the rising transport and mobility demand [10]. For instance, in the EU, road transport contributes to $24.2 \%$ of energy consumption, $16.7 \%$ of greenhouse gas (GHG) emissions, and more than 25,000

\footnotetext{
*Correspondence: mmakridis@ethz.ch

2European Commission - Joint Research Centre, Directorate for Energy,

Transport and Climate Change, Ispra, VA, Italy

Full list of author information is available at the end of the article
}

deaths each year [6, 37]. Motivated to solve these problems, advanced driver assistance systems (ADAS) gain traction among automakers and consumers. Adaptive cruise control (ACC), as one of the most widely used ADAS features, can automatically adjust the vehicle speed to maintain a userspecified time headway, or reach the speed desired by the user. However, surging acceptance of ACC across the globe is further escalating concerns over its energy impact in realworld scenarios, due to its ability to govern the vehicle 
longitudinal driving behaviour for extended periods of distance and time.

The launching of ACC onto the market has triggered a proliferation of innovative studies that examine ACC driving behaviour in real-world scenarios, such as higher than expected response time and time headway values comparable to human drivers [23, 24]. Recent advances in on-board measuring and high-performance computing have facilitated investigations on the energy impact of ACC driving behaviour [31]. For example, the simulation study of an ACC-equipped heavy-duty truck claimed to achieve fuel savings of $5.9 \%$ and $2.2 \%$ during urban and highway driving scenarios, respectively [18]. In another major study, ACC driving was reported to deliver fuel savings of $22-31 \%$ and $12-26 \%$ within acceleration and deceleration phases, respectively [34]. It was also demonstrated that the ACC system could simultaneously improve fuel efficiency and traffic flow [25].

ACC operates in the two driving modes of free-flow and car-following. Accordingly, the studies on the energy impact of ACC driving behaviour can be divided into following two broad groups:

- The studies for free-flow scenarios usually overlook the interactions with surrounding vehicles. Instead, the road speed limit, the location of traffic signals and the roadway topography, etc., are used to compute the most energy-efficient velocity profile $[9,14$, $22,30,34]$. For instance, an ACC system was proposed to minimize fuel consumption and travel time, using the known road topography and the Dijkstra's algorithm [32]. In addition, Porsche Innodrive ACC [26] adopted a similar technique with precise knowledge of vehicle specifications and the route ahead, claimed to deliver energy savings of $10 \%$ on average.

- The studies for car-following scenarios mainly focus on the energy impact of ACC driving behaviour during vehicle interactions [11, 19, 20, 36]. For example, an ACC system was developed to keep a certain headway distance to the vehicle ahead and could achieve the fuel economy benefit of $12.4 \%$ in car-following tests [20].

How to compute or measure energy consumption is vitally important for assessing the energy impact of ACC. It is worth noting that the propulsion system, vehicle specifications, and road and traffic conditions also have significant effects on energy consumption [8]. There are four energy estimation methods often observed in the literature:

- In the measured-data-based method, the instantaneous fuel consumption rate is calculated using the mass air flow (MAF) signal read from vehicle OBDII (on-board diagnostics II) [15]. However, this method has two assumptions causing a slight deviation from the true value: the fuel density is fixed to $720 \mathrm{~g} / \mathrm{l}$ and the air-fuel ratio is fixed to 14.7 [30].

- In the mathematical-model-based method, the physical process of powertrain dynamics is described by mathematical equations $[17,33,35]$. It is a widely used approach for the vehicle with a hybrid propulsion system. For example, in the fleet consisting of multiple power-split hybrid electric vehicles (HEVs), the cooperative driving control and the powertrain energy management were co-optimized to deliver an energy saving of $17.9 \%$ [21].

- In the regression-model-based method, the regression model is fitted to data from laboratory tests and then used to predict fuel consumption by taking the vehicle's speed and acceleration as inputs $[1,7,38]$.

- In the powertrain-free-based method, the energy indicator only considers the tractive power demand (positive) placed at the wheels $[4,13]$, while the powertrain dynamics and the regenerative braking power (negative) are not taken into account.

Most of the prior studies are carried out in numerical simulations or test-track experiments. To the best of the authors' knowledge, only a limited number of investigations contribute to understanding the energy impact of ACC driving behaviour in real-world applications. A large-scale study with a fleet of 51 test vehicles over 62 days and 199,300 miles (driven by General Motor employees on their daily commutes) was conducted to analyse the GHG emissions benefit of ACC from a statistical perspective [5]. It's important to stress that GHG emissions are directly correlated to fuel consumption. The results showed that ACC driving could significantly reduce GHG emissions at low speeds, which, however, would hardly deliver a meaningful benefit for the whole journey, because ACC utilization rates at low speeds were marginal and low-speed driving only covered a small portion of the total travel distance. Another field experiment with five vehicles was reported [30] to compare the free-flow driving behaviour between cruise control systems and human drivers. The data demonstrated that cruise control could reduce fuel consumption by $3.3 \%$ on average relative to human driving. So far, however, no research has been found that surveyed ACC energy impact in real-world car-following scenarios. The current research sets out to fill this gap.

Two questions have directed much of this project: how to distinguish ACC driving behaviour from that of the human driver and how to identify the ACC energy impact. As opposed to simulations or test-track experiments as described in previous studies, this work is unique because it was performed in real-world carfollowing scenarios with a variety of vehicle 
specifications, propulsion systems, drivers, and road and traffic conditions. Tractive energy consumption serves as the energy impact indicator, ruling out the effect of the propulsion system. To further isolate the driving behaviour as the only possible contributor to tractive energy differences, two techniques are offered to normalize heterogeneous vehicle specifications and road and traffic conditions. Finally, ACC driving behaviour is compared with that of the human driver from transient and statistical perspectives. Its impact on tractive energy consumption is then evaluated from individual and platoon perspectives. Our data suggest that unlike human drivers, ACC followers lead to string instability, i.e., amplifying downstream speed variations. Their inability to absorb the speed overshoots may partly be explained by their high responsiveness. Statistical results might imply the followers in the automated or mixed traffic flow generally perform worse in reproducing the driving style of the preceding vehicle. On the individual level, ACC followers have tractive energy consumption 2.7$20.5 \%$ higher than those of human counterparts. This finding is contrary to a recent study by General Motor [5] because it fails to exclude the fuel-efficient ACC free-flow driving. On the platoon level, the tractive energy values of ACC followers tend to consecutively increase $(11.2-17.3 \%)$ as the speed perturbations propagate upstream. In general, therefore, ACC impacts negatively on tractive energy efficiency. This research provides a feasible path for evaluating the energy impact of ACC in real-world applications. Moreover, the findings have significant implications for ACC safety design when handling the stability-responsiveness trade-off.

The rest of the paper is organized as follows. Section 2 describes the proposed methodology, including the modelling of tractive energy demand and the experimental data acquisition campaign. In Section 3, the results are discussed from the perspectives of driving behaviour and tractive energy consumption. Section 4 concludes the paper by summarizing the main findings and implications in this work.

\section{Methods}

\subsection{Modelling of tractive energy consumption}

When approaching the energy impact of ACC driving behaviour, the aforementioned powertrain-free-based tractive energy consumption is a more suitable assessment indicator from a transport engineering perspective. Although it doesn't directly reflect the engine fuel consumption or the battery charge depletion, this metric can rule out the energy effect of heterogeneous propulsion systems in the traffic network. In addition, the auxiliary loads are not taken into account in the computation of tractive energy consumption, but all test vehicles were using the same auxiliary functions during the driving campaign in this study. Specifically, the vehicle's tractive energy consumption $\left(E_{\mathrm{t}}, \mathrm{kWh} / 100 \mathrm{~km}\right)$ is calculated by integrating the tractive power requirements $\left(P_{\mathrm{t}}, \mathrm{kW}\right)$ at the wheels over time, not considering the negative power components from the regenerative braking, as described by:

$$
\begin{aligned}
P_{t} & = \begin{cases}\left(F_{0}+F_{1} v_{e}+F_{2} v_{e}^{2}+1.03 m a_{e}+m g \cdot \sin \theta\right) v_{e} \cdot 10^{-3}, & P_{t} \geq 0 \\
0, & P_{t}<0\end{cases} \\
E_{t} & =\frac{\int_{0}^{T} P_{t} d t}{0.036 \cdot \int_{0}^{T} v_{e} d t},
\end{aligned}
$$

where $F_{0}, F_{1}$ and $F_{2}$ are road load coefficients; $m$ is the vehicle mass $(\mathrm{kg}) ; v_{e}$ and $a_{e}$ are the ego vehicle's speed $(\mathrm{m} / \mathrm{s})$ and acceleration $\left(\mathrm{m} / \mathrm{s}^{2}\right)$, respectively; $\theta$ is the road gradient (rad); $g$ is the gravitational acceleration $(9.81 \mathrm{~m} /$ $\mathrm{s}^{2}$ ); $d t$ is the time interval (s) between consecutive measurement points; $T$ denotes the total duration (s) of the travel period.

There are six broad categories of factors that influence tractive energy consumption [2], namely, driving-, vehicle-, road-, traffic-, travel- and weather-related. As illustrated in Fig. 1, this study captures the first four major factors [13]: i) driving-related, i.e., ACC or human driving behaviour, mainly characterized by the ego vehicle's velocity and acceleration profiles; ii) vehicle-related, indicating vehicle specifications such as operation mass and air drag coefficient; iii) road-related, e.g., road gradient; and iv) traffic-related, including the inter-vehicle distance and the relative speed, etc., which can describe the ego vehicle's interaction with surrounding vehicles.

\subsection{Experimental design}

Figure 2 represents the real-world experiment in highway multiple-car-following scenarios. Field tests were conducted on a section of Autostrada A26 (Italy) between Ispra and Vicolungo, a $124.6 \mathrm{~km}$ round trip, to collect driving data under actual traffic conditions. Five vehicles were equipped with five identical, commercial, multi-constellation GNSS receivers, able to collect GNSS data with a $10 \mathrm{~Hz}$ measurement rate. The receivers were configured to collect signals from both GPS and Galileo, the European GNSS, with the ability to process up to 16 satellite signals, enabling good performance (a minimum of 4 satellite signals are required for positioning using a single constellation). The average horizontal accuracy reported by the receivers was less than $50 \mathrm{~cm}$. GNSS active antennas were mounted on the roof of the cars, in order to ensure maximum satellite visibility and avoid signal attenuations from the body of the vehicles. At each time instant, the geographic coordinates (latitude, longitude and altitude) of the vehicles were recorded. These 


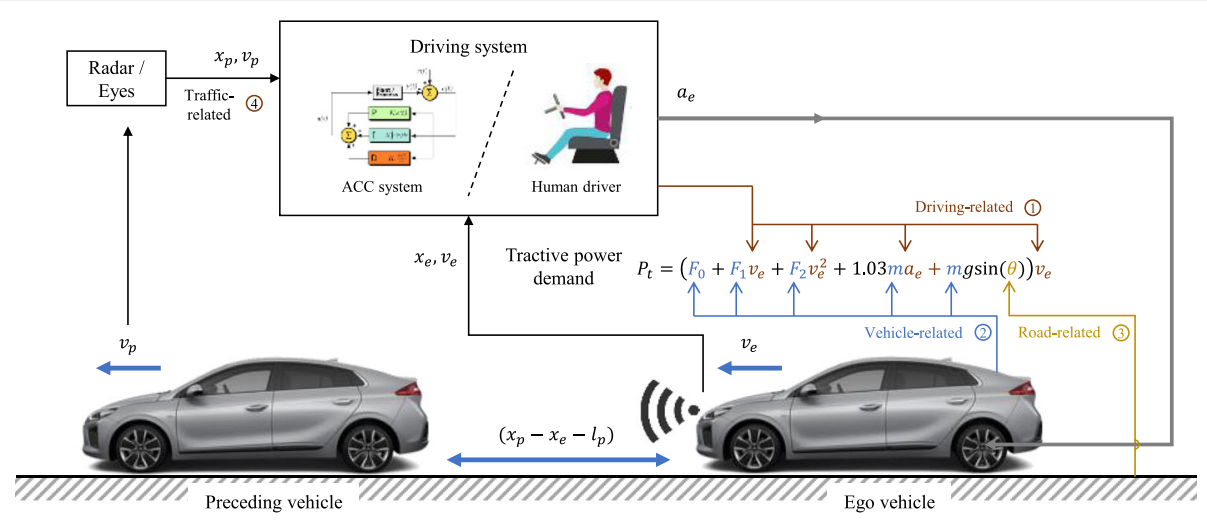

Fig. 1 The tractive power demand at the wheels

coordinates were then transformed into a local East, North and Up (ENU) Cartesian reference frame. Outliers were filtered using typical moving average postprocessing. The test section comprises various uphill and downhill segments, providing an appropriate environment to examine different tractive load conditions for human or ACC driving. The elevation profile of the test route was extracted using Google API, as shown in Fig. 2a and b. The road gradient varies approximately between $-8 \%$ and $8 \%$, where some rather steep points appear since the highway section is located at the foot of the Alps Mountains.

Table 1 summarizes the specifications of five test passenger cars, including the leader $(\mathrm{C} 1)$ and four followers $(\mathrm{C} 2, \mathrm{C} 3, \mathrm{C} 4$ and $\mathrm{C} 5)$. These vehicles were selected to test different manufacturers, models, years and types. As explained in Fig. 2, on the southbound (SB) route (from Ispra to Vicolungo), all vehicles were operated by human drivers. On the northbound (NB) route, however, the followers (C2, C3 and C4) adopted ACC driving to regulate the longitudinal speed and the inter-vehicle distance.
While their leader (C1) and the last one (C5) were human-driven, whose drivers were respectively the same as those on the SB route.

The multiple-car-following tests were not replicated, owing to the impossibility of replicating the traffic conditions in real-world scenarios, but our driving tests sampled a wide range of variations in highway traffic. It is worth noting that some unexpected issues (e.g., the interference of cut-in vehicles and a brief GPS signal lost when passing through a tunnel) are unavoidable in the real-world car-following experiments. To deal with these challenges and yield meaningful results, if any of these issues occur, that piece of trajectory is ignored for all test vehicles in the computation of tractive energy consumption. However, these parts only covered a small portion of the total trajectory in our car-following campaign. In addition, the data points included in the analyses of this study have the acceleration ranging from -5 to $3 \mathrm{~m} / \mathrm{s}^{2}$ to rule out data that are generally considered to come from GNSS measurement errors. The outliers beyond this acceleration range appear in each of the

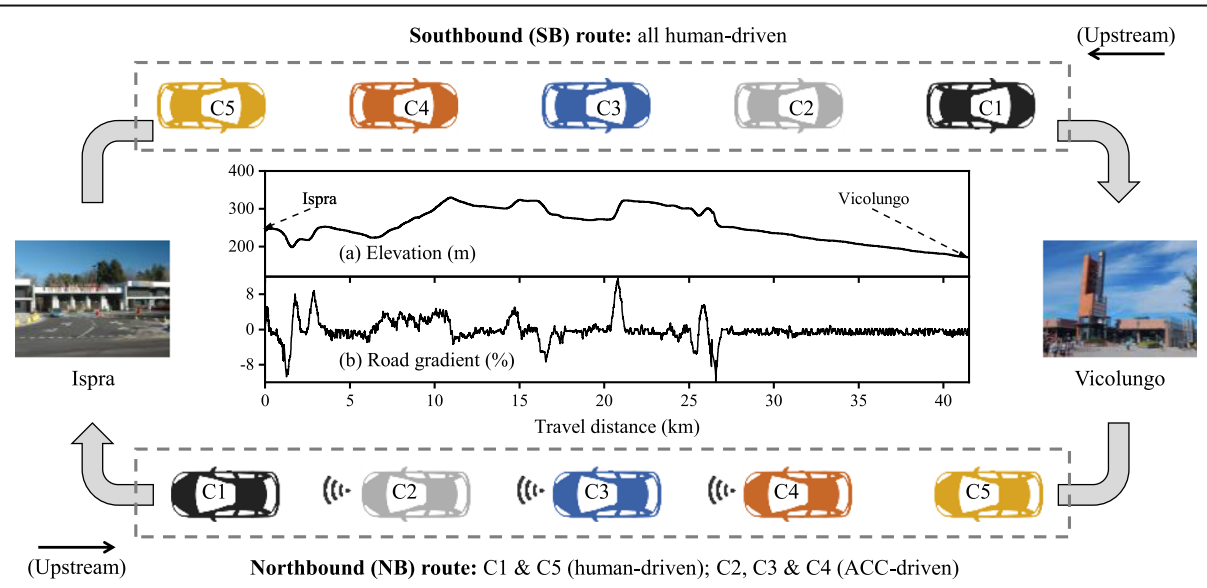

Fig. 2 The real-world driving experiment in highway multiple-car-following scenarios 
Table 1 Specifications of the test vehicles

\begin{tabular}{llllllll}
\hline Cars & Release year & Car type & Transmission & Mass $(\mathrm{kg})$ & Width $(\mathrm{m})$ & Height $(\mathrm{m})$ & Max. power $(\mathrm{kW})$ \\
\hline C1 & 2001 & $\mathrm{mpv}$ & automatic & 1430 & 1.715 & 1.515 & 72 \\
$\mathrm{C2}$ & 2018 & $\mathrm{mpv}$ & automatic & 1838 & 1.884 & 1.658 & 120 \\
$\mathrm{C} 3$ & 2009 & $\mathrm{mpv}$ & automatic & 1764 & 1.837 & 1.639 & 110 \\
$\mathrm{C} 4$ & 2017 & suv & automatic & 1650 & 1.805 & 1.545 & 104 \\
$\mathrm{C5}$ & 2011 & coupe & manual & 1315 & 1.683 & 1.378 & 90 \\
\hline
\end{tabular}

real-world driving trajectories but only account for a small proportion (less than 2.5\%).

\section{Results and discussions}

This section employs several data analysis techniques to present the results. Firstly, ACC driving behaviour is compared with that of the human driver from transient and statistical perspectives. In the transient analysis, speed and tractive power profiles are closely examined to quantify the string stability and the braking response time, respectively. While the statistical analysis is conducted to explore the joint and marginal distributions of speed, acceleration and tractive power of each test vehicle in the campaign. Secondly, the impact of ACC on tractive energy consumption is evaluated from individual and platoon perspectives. To isolate the driving behaviour as the only possible contributor to tractive energy differences, the following two techniques are offered to normalize heterogeneous vehicle specifications and road and traffic conditions.

- It is challenging to assess the ACC tractive energy impact on the individual level, namely, to compare the tractive energy values of the same vehicle (e.g., $\mathrm{C} 2, \mathrm{C} 3$ or $\mathrm{C} 4$ in Fig. 2) driven by the ACC system and the human driver (on NB and SB routes, respectively). The main reason for this issue is that ACC and the human driver experience different road and traffic conditions on different routes. To draw meaningful comparisons, the technique denoted as individual normalization, divides vehicles' tractive energy consumption $\left(E_{t, \mathrm{Ci}}\right)$ by that of the leading one $\left(E_{t, \mathrm{Cl}}\right)$ on the same route, resulting in individual normalized tractive energy consumption $E_{t, \mathrm{Ci}}^{i n}$ for each vehicle (Ci).

- On the other hand, ACC tractive energy impact on the platoon level is assessed by comparing the tractive energy values of vehicles in the same platoon (e.g., C1-C2-C3-C4-C5 on the NB route). Although the vehicles in the same platoon experience nearly the same road and traffic conditions, their specifications are heterogeneous. To tackle this challenge, the technique denoted as platoon normalization assumes that all the vehicles on the same route share the same specifications as those of their leader (C1) and compute the platoon normalized tractive energy consumption $E_{t, \mathrm{Ci}}^{p n}(\mathrm{kWh} / 100$ $\mathrm{km}$ ) for each vehicle (Ci).

\subsection{Transient analysis of driving behaviour}

To analyse the transient driving behaviours in the highway multiple-car-following scenario, Fig. 3 provides speed/acceleration/tractive power vs time coordinate graphs for test vehicles. Specifically, a string of 5 passenger cars driven by the human driver or the ACC system moves from Ispra to Vicolungo (southbound, SB) and vice versa (northbound, NB) in a fixed order. As time goes by, the leader ( $\mathrm{C} 1)$ decelerates or accelerates according to the traffic conditions. We can then compare different responses of the rest four followers and compute their corresponding tractive power according to Eq. (1).

In previous studies, string stability is usually defined as the phenomenon that the leader's speed variations are not amplified through the upstream followers [16]. On the SB route, two grey sections (time intervals 130-180 s and $1120-1170 \mathrm{~s})$ in Fig. 3a and e are zoomed in and put into the same speed/acceleration vs time coordinates in Fig. 3f-i. The black curved arrows in Fig. $3 \mathrm{f}$ and $\mathrm{h}$ indicate the stable car-following behaviour of human drivers. It is clear that steep speed variations of the leader $(\mathrm{C} 1)$ are not amplified through the human-driven followers (C2, C3, C4, and C5), whose velocities are not obviously fluctuated. On the NB route, by contrast, two selected time intervals (200-250 s and 1230-1280 s) shown in Fig. $3 \mathrm{p}-\mathrm{s}$ highlight the unstable ACC carfollowing behaviours. ACC followers (C2, C3, and C4) significantly enlarge their leader's speed perturbations propagating upstream. Thus, their inter-vehicle distances also fluctuate, causing rear-end collision safety concern. However, the last follower (C5), operated by the human driver, doesn't exacerbate the instability propagating upstream, and sometimes even can reduce this perturbation.

To quantitatively assess the amplification of speed perturbations in the car platoon, the percentage overshoot ( $\left.M_{\mathrm{Ci}}^{p}\right)$ serves as the indicator $[28,29]$, which can be expressed as: 


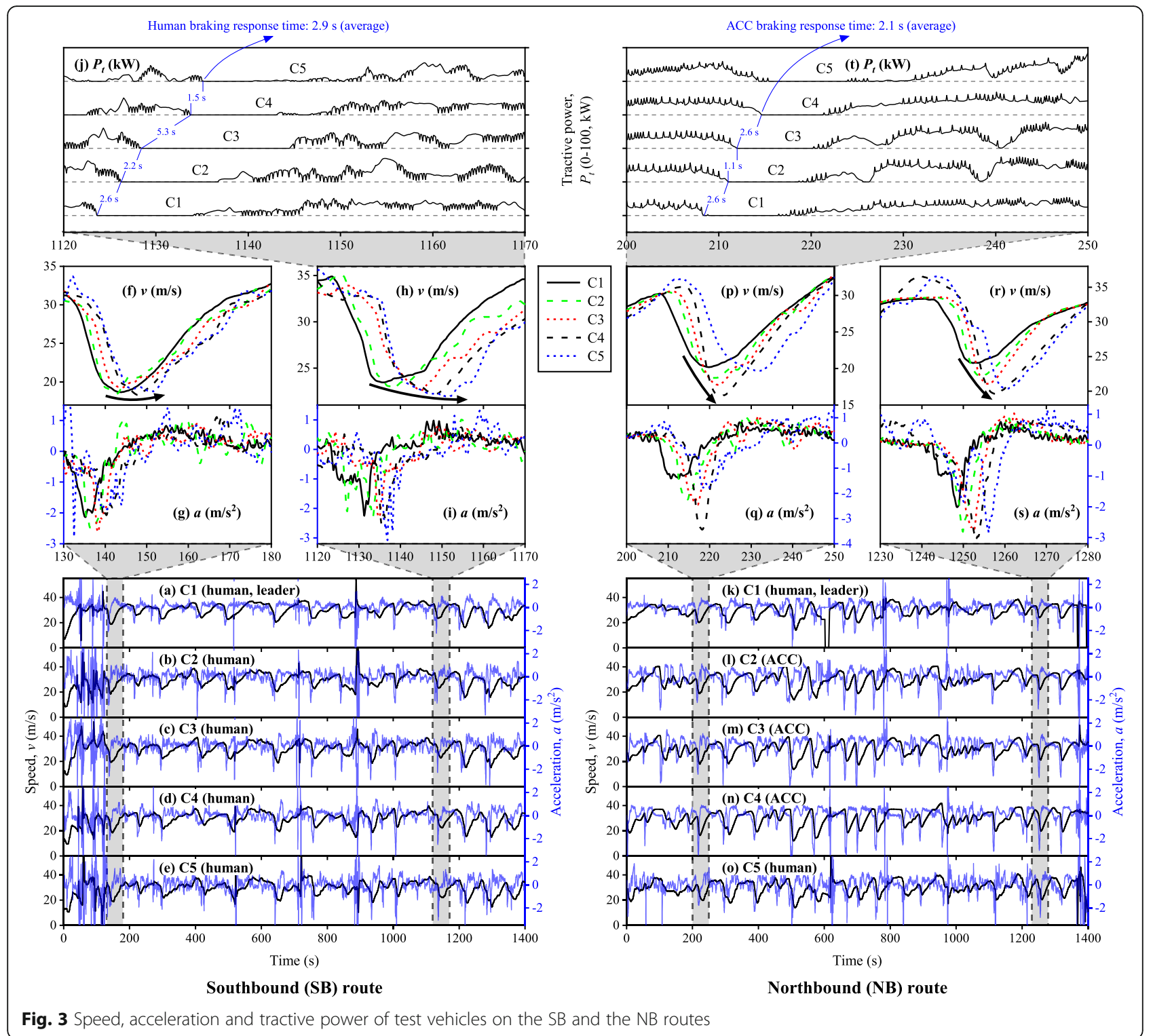

$$
M_{C i}^{p}=\left(\frac{\max \left(v_{C i}\right)-\min \left(v_{C i}\right)}{\max \left(v_{C 1}\right)-\min \left(v_{C 1}\right)}-1\right) \times 100 \%,
$$

Figure 4 compares the percentage overshoots of test vehicles on both routes within the selected time intervals. It is apparent that there are no obvious trends for the speed overshoots of human followers on the SB route (blue). Their $M_{\mathrm{Ci}}^{p}$ values lie around $0 \%$. When we examine ACC followers on the NB route (black), however, strong growth trends in their $M_{\mathrm{Ci}}^{p}$ values are detected. Their percentage overshoots vary from $\sim 15 \%$ to $\sim 31 \%$ in the $200-250 \mathrm{~s}$ interval, and from $\sim 24 \%$ to $83 \%$ in the $1230-1280 \mathrm{~s}$ interval. It is important to stress that the last follower ( $\mathrm{C} 5$, human-driven) on the NB route can mitigate these upward trends. The quantitative evidence here is consistent with the above findings in

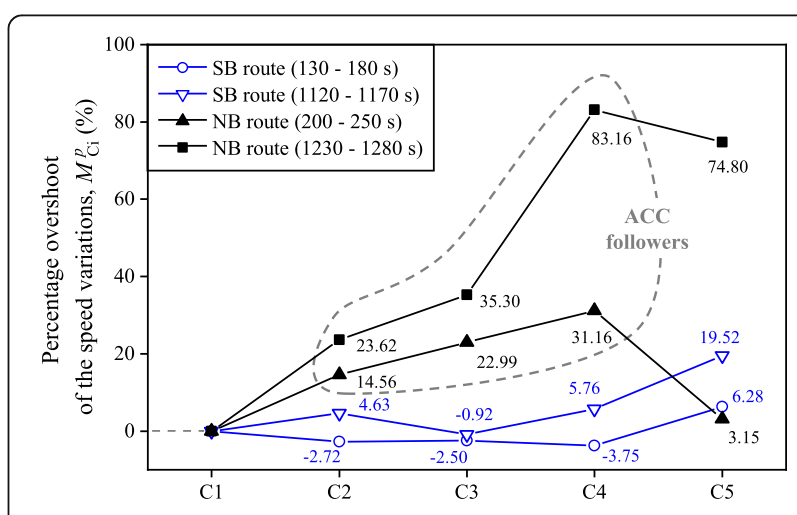

Fig. 4 The percentage overshoots of speed variations in the platoon 
Fig. 3, namely, consecutive ACC followers can cause string instability, and human followers, however, can react in a way to mitigate speed perturbations propagating upstream.

Figure $3 j$ and $t$ illustrate the tractive power profiles of test vehicles during the SB (1120-1170s) and the NB (200-250 s) trips, respectively. The vertically stacked tractive power curves are plotted in five horizontal bands, each of which ranges from 0 to $100 \mathrm{~kW}$. In these charts, the tractive power only has positive components and remains zero during braking manoeuvres. By comparing the starting points of the braking phases, we can identify the braking response time of each follower. Figure 3j shows that the human drivers' average braking response delay is $2.9 \mathrm{~s}$ in the selected time interval. As for ACC tractive power profiles presented in Fig. 3t, the average braking response time is $2.1 \mathrm{~s}$. The results, in this case, report that the responsiveness of ACC is on average higher than that of the human driver. This may partly explain the ACC instability car-following performance shown above because as stated in previous studies $[3,12]$, there exists a trade-off between stability and responsiveness in the control system design and calibration. Namely, the faster the control system response, the more unstable the speed tracking (or the more significant the overshoot).

\subsection{Statistical analysis of driving behaviour}

Figure 5 uses contour heatmaps to illustrate the speedacceleration joint probability distributions of the vehicles. The similarity between the distribution heatmaps of two consecutive vehicles is quantified with the 2D correlation coefficient (R) [27]. The similarity coefficients demonstrate that in human-to-human car-following scenarios (on the $\mathrm{SB}$ route), the $\mathrm{R}$ values lie between 0.86 and 0.88 , generally greater than their human-to-ACC, ACC-to-ACC or ACC-to-human counterparts (on the NB route). The finding reported here might imply that the followers in the automated or mixed traffic flow, relative to those in the manual traffic, generally perform worse in reproducing the driving style of the immediately preceding vehicle. The likely cause for this difference is that the ACC system mainly aims to keep a safe time headway thus shows less flexibility.

Box-and-whisker diagrams shown in Fig. 6 present marginal distributions of tractive power, speed, and acceleration. The height of the box is the interquartile range (IQR) between the first quartile $\left(\mathrm{Q}_{1}, 25 \%\right)$ and the third quartile $\left(\mathrm{Q}_{3}, 75 \%\right)$, namely, $\mathrm{IQR}=\mathrm{Q}_{3}-\mathrm{Q}_{1}$. The median, the band inside the box, is the second quartile $\left(\mathrm{Q}_{2}, 50 \%\right)$. The ends of the whisker $\left(\mathrm{Q}_{1}-1.5 \mathrm{IQR}\right.$ and $\left.\mathrm{Q}_{3}+1.5 \mathrm{IQR}\right)$ represent the outlier-free interval (OFI).

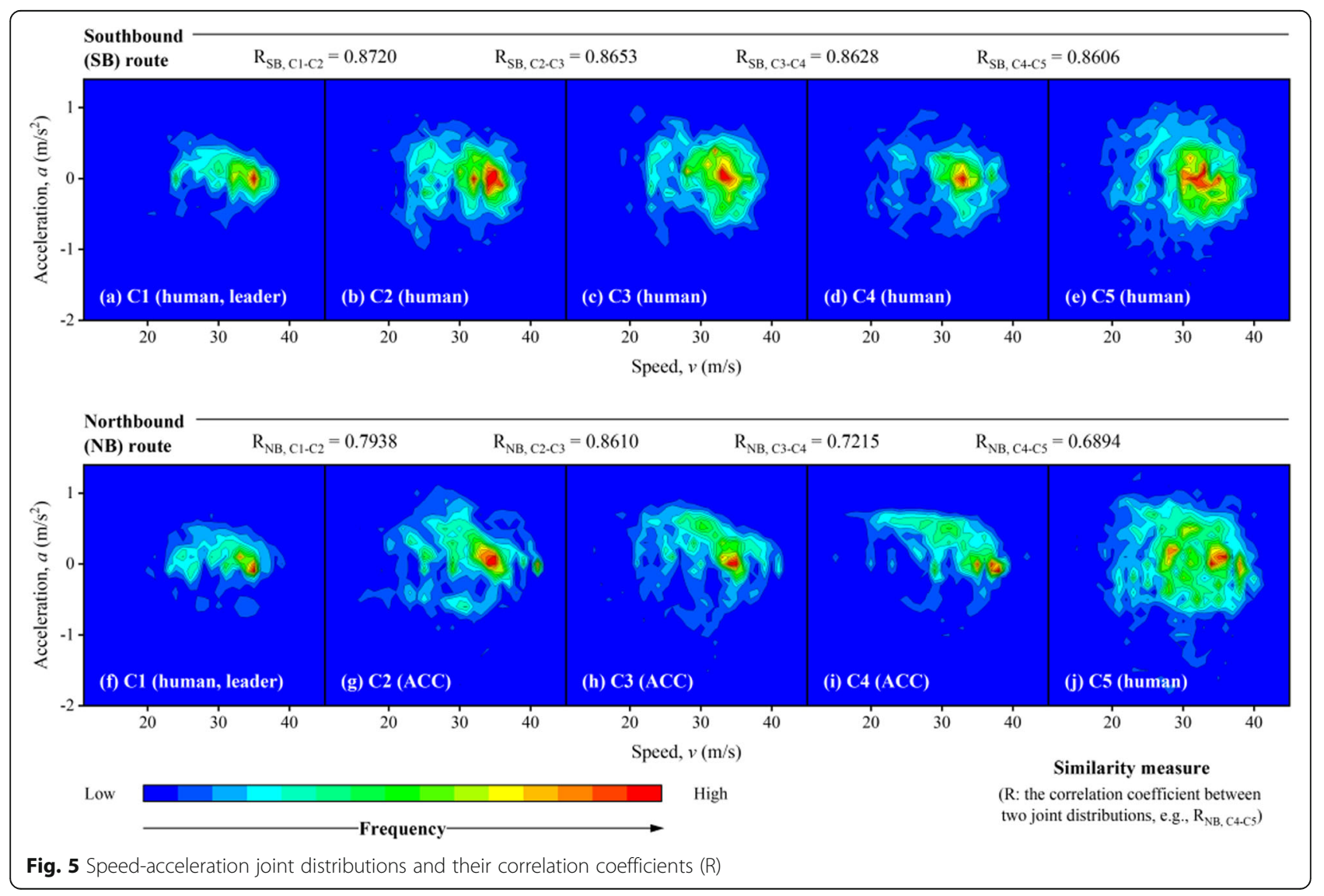




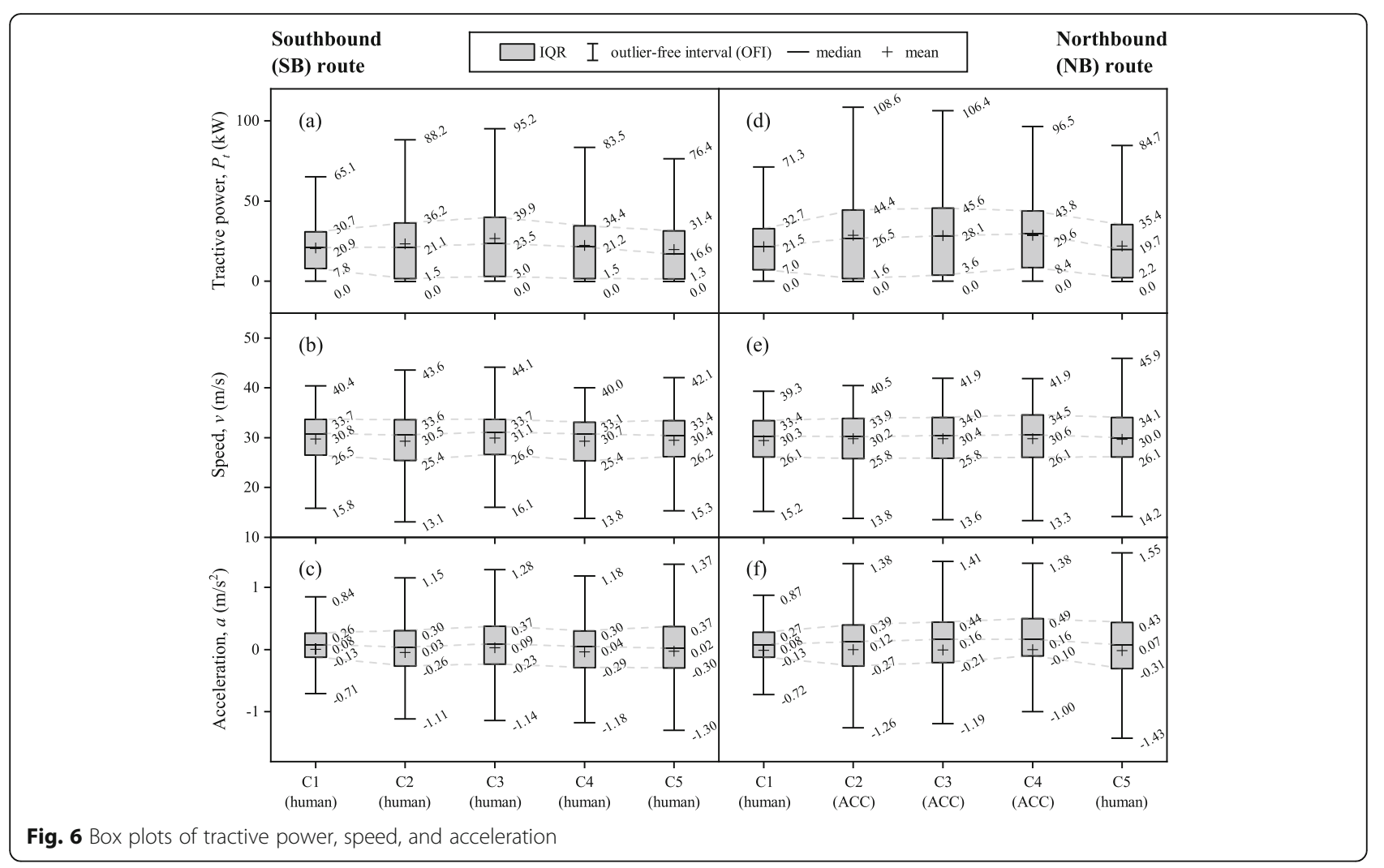

Data points beyond this interval are viewed as being too far from the central value to be reasonable. It should be noted that the upper boundaries of tractive power OFIs in Fig. $6 \mathrm{a}$ and d are all compliant with the test vehicles' maximum power in Table 1, demonstrating the reliability and validity of test measurements and mathematical modelling in this study. In addition, the distributions in Fig. $6 \mathrm{~b}$ and e show that the speed values in this highway scenario vary from 13.1 to $45.9 \mathrm{~m} / \mathrm{s}$, and their medians lie between 30.0 and $31.1 \mathrm{~m} / \mathrm{s}$.

Table 2 summarizes the interquartile range of speed and acceleration distributions. Closer inspection of the acceleration columns $\left(\mathrm{IQR}_{a}\right)$ shows that ACC- and human-driven followers all have a greater $\mathrm{IQR}_{a}$ than that of their leader $(\mathrm{C} 1)$. What stands out in speed columns $\left(\mathrm{IQR}_{v}\right)$ is that $\mathrm{ACC}$ followers (the bold numbers) ramp

Table 2 Interquartile range (IQR) of speed ( $v$ ) and acceleration (a) distributions

\begin{tabular}{|c|c|c|c|c|c|c|}
\hline & \multicolumn{3}{|c|}{ Southbound (SB) } & \multicolumn{3}{|c|}{ Northbound (NB) } \\
\hline & Driving & $\mathrm{IQR}_{v}$ & $\mathrm{IQR}_{a}$ & Driving & $\mathrm{IQR}_{V}$ & $\mathrm{IQR}_{a}$ \\
\hline C1 & human & 7.2 & 0.39 & human & 7.3 & 0.40 \\
\hline C2 & human & 8.2 & 0.58 & ACC & 8.1 & 0.66 \\
\hline C3 & human & 7.1 & 0.62 & ACC & 8.2 & 0.65 \\
\hline C4 & human & 7.7 & 0.60 & ACC & 8.4 & 0.59 \\
\hline $\mathrm{C} 5$ & human & 7.2 & 0.67 & human & 8.0 & 0.74 \\
\hline
\end{tabular}

up the driving speed dispersion, however, the human followers can sometimes reduce $\mathrm{IQR}_{v}$ (the underlined numbers). This finding is consistent with the above discussions, which demonstrate that human driving may decrease speed perturbations propagating upstream.

\subsection{ACC energy impact on the individual level}

As previously stated, the individual normalized tractive energy consumption $\left(E_{t, \mathrm{Ci}}^{i n}\right)$ is computed by dividing each vehicle's tractive energy consumption $\left(E_{t, \mathrm{Ci}_{\mathrm{i}}}\right)$ by that of the leading one $\left(E_{t, \mathrm{C} 1}\right)$ on the same route:

$$
E_{t, C i}^{i n}=\frac{E_{t, C i}}{E_{t, C 1}}
$$

Figure 7 shows $E_{t, \mathrm{Ci}}^{i n}$ values of test vehicles on the $\mathrm{SB}$ and the NB routes, also provides the percentage $E_{t, \mathrm{Ci}}^{i n}$ increase caused by ACC driving. The grouped bars of the last follower (C5) operated by the same human driver, suggest that there is an error of about $6 \%$ when evaluating ACC energy impact using the proposed individual normalization technique. The results from test vehicles C2, C3 and C4 demonstrate that ACC followers have tractive energy consumption $2.7-20.5 \%$ higher than those of human counterparts. This may be attributed to string instability and high responsiveness of ACC systems as discussed in Figs. 3 and 4. This outcome is 


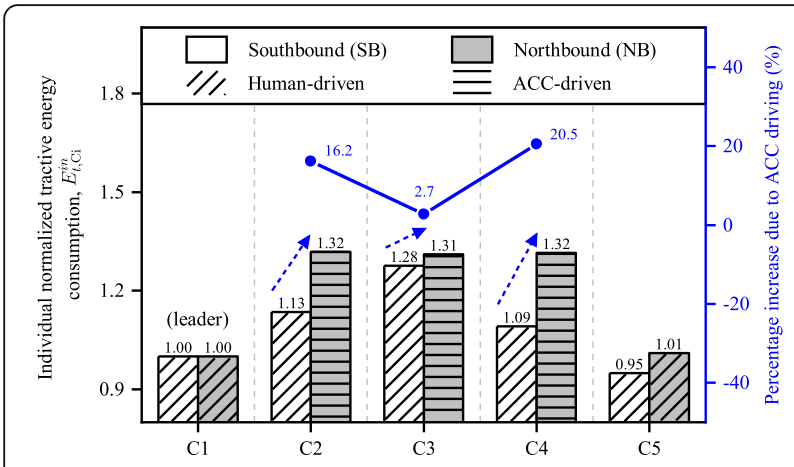

Fig. 7 ACC energy impact on the individual level

contrary to a recent study by General Motor [5] that reported a positive total GHG (directly correlated to fuel consumption) emissions benefit of the ACC system. The most likely cause of this controversy is that Dvorkin's analysis also takes account of fuel-efficient ACC freeflow driving, which can significantly reduce the unnecessary acceleration/braking by human drivers [30].

\subsection{ACC energy impact on the platoon level}

The platoon normalized tractive energy consumption ( $\left.E_{t, \mathrm{Ci}}^{p n}\right)$ assesses ACC energy impact on the platoon level by assuming that all the vehicles on the same route share the same specifications as those of their leader (C1). The marked lines in Fig. 8a illustrate the $E_{t, \mathrm{Ci}}^{p n}$ values of test vehicles in the homogenized platoon. On the $\mathrm{SB}$ route (blue), their values range from 18.84 to $21.56 \mathrm{kWh} / 100 \mathrm{~km}$. Meanwhile, on the NB route (black), in the platoon mixed with ACC- and human-driven vehicles, the $E_{t, \mathrm{Ci}}^{p n}$ values lie between 20.17 and $23.66 \mathrm{kWh} /$ $100 \mathrm{~km}$. Figure $8 \mathrm{~b}$ shows the followers' percentage $E_{t, \mathrm{Ci}}^{p n}$

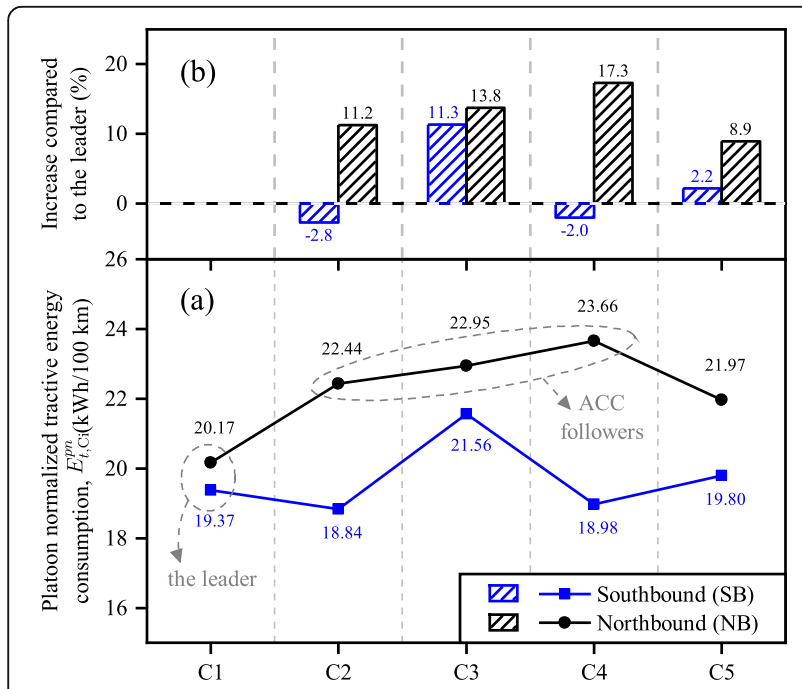

Fig. 8 ACC energy impact on the platoon level increase compared with that of their leader. It is apparent from this chart that the tractive energy values of ACC followers tend to consecutively increase (11.2$17.3 \%$ ) along with speed perturbations propagating upstream, as emphasized in Fig. 4. Therefore, the negative effect of ACC on tractive energy efficiency is likely to be related to its string instability characteristic.

\section{Conclusions}

Returning to the questions posed at the beginning of this study, it is now possible to state that the proposed methods can effectively distinguish ACC driving behaviour from that of the human driver and identify the ACC energy impact, in real-world scenarios with a variety of vehicle specifications, propulsion systems, drivers, and road and traffic conditions. Tractive energy consumption serves as the energy impact indicator that can rule out the effect of the propulsion system. To further isolate the driving behaviour as the only possible contributor to tractive energy differences, the individual and the platoon normalization techniques are offered to homogenize vehicle specifications and road and traffic conditions. Finally, ACC driving behaviour is compared with that of the human driver from transient and statistical perspectives. Its impact on tractive energy consumption is then evaluated from individual and platoon perspectives. The results from the above methods suggest that:

- ACC followers lead to string instability, i.e., amplifying downstream speed variations. However, human drivers can react in a way to mitigate these speed perturbations.

- The inability of ACC to absorb the speed overshoots may partly be explained by their high responsiveness.

- Statistical results might imply the followers in the automated or mixed traffic flow, relative to those in the manual traffic, generally perform worse in reproducing the driving style of the immediately preceding vehicle.

- On the individual level, ACC followers have tractive energy consumption $2.7-20.5 \%$ higher than those of human counterparts.

- On the platoon level, the tractive energy values of ACC followers tend to consecutively increase (11.2$17.3 \%)$ as the speed perturbations propagate upstream.

In general, therefore, ACC impacts negatively on tractive energy efficiency. This research can provide a feasible path for evaluating the energy impact of ACC in real-world applications. Furthermore, these findings have significant implications for ACC safety design, 
particularly for handling the stability-responsiveness trade-off. A future study carried out on test tracks would be instrumental in our further understanding of ACC energy impacts.

\section{Acknowledgements}

This work was based on a collaboration agreement between the Joint Research Centre of the European Commission and the University of Birmingham. The authors are grateful to Eleftherios-Nektarios Grylonakis and Vincenzo Arcidiacono who contributed to the data processing as well as to Fabrizio Minarini, Norbert Brinkhoff-Button, Daniele Borio, Alessandro Tansini, Aikaterini Anesiadou and Melania Susi for their support during the experimental campaign

\section{Authors' contributions}

MM, BC and GF coordinated the study and designed the methodology. KM and $\mathrm{YH}$ collected the data and performed the data analysis. The draft manuscript preparation was made by $\mathrm{YH}$ and $\mathrm{MM}$. BC, GF and HX contributed to the writing of the manuscript. The authors read and approved the final manuscript.

\section{Funding}

Not applicable.

\section{Availability of data and materials}

Not applicable.

\section{Competing interests}

The authors declare that they have no competing interests.

\section{Author details}

${ }^{1}$ University of Birmingham, Edgbaston, Birmingham B15 2TT, UK. ${ }^{2}$ European Commission - Joint Research Centre, Directorate for Energy, Transport and Climate Change, Ispra, VA, Italy.

Received: 5 July 2019 Accepted: 25 February 2020 Published online: 24 March 2020

\section{References}

1. Ahn, K. (1998). Microscopic fuel consumption and emission modeling (thesis) Virginia Tech.

2. Ahn, K., Rakha, H., Trani, A., \& Van Aerde, M. (2002). Estimating vehicle fuel consumption and emissions based on instantaneous speed and acceleration levels. Journal of Transportation Engineering, 128, 182-190.

3. Bedner, E., Fulk, D., \& Hac, A. (2007). Exploring the trade-off of handling stability and responsiveness with advanced control systems (SAE technical paper no. 2007-01-0812). Warrendale: SAE International. https://doi.org/10. 4271/2007-01-0812

4. Ciuffo, B., Makridis, M., Toledo, T., \& Fontaras, G. (2018). Capability of current car-following models to reproduce vehicle free-flow acceleration dynamics. IEEE Transactions on Intelligent Transportation Systems, 19, 3594-3603.

5. Dvorkin, W., King, J., Gray, M., \& Jao, S. (2019). Determining the greenhouse gas emissions benefit of an adaptive cruise control system using real-world driving data. Presented at the WCX SAE World Congress Experience. Detroit.

6. European Commission. (2016). A European strategy for low-emission mobility.

7. Faris, W. F., Rakha, H. A., Kafafy, R. I., Idres, M., \& Elmoselhy, S. (2011). Vehicle fuel consumption and emission modelling: An in-depth literature review. International Journal of Vehicle Systems Modelling and Testing, 6, 318-395.

8. Fiori, C., Arcidiacono, V., Fontaras, G., Makridis, M., Mattas, K., Marzano, V. Thiel, C., \& Ciuffo, B. (2019). The effect of electrified mobility on the relationship between traffic conditions and energy consumption. Transportation Research Part D: Transport and Environment, 67, 275-290.

9. Hellström, E., Åslund, J., \& Nielsen, L. (2010). Design of an efficient algorithm for fuel-optimal look-ahead control. Control Engineering PracticeSpecial Issue on Automotive Control Applications, 2008 IFAC World Congress, 18, 13181327.

10. Jia, D., Lu, K., Wang, J., Zhang, X., \& Shen, X. (2016). A survey on platoonbased vehicular cyber-physical systems. IEEE Communication Surveys and Tutorials, 18, 263-284.
11. Kamal, M. A. S., Mukai, M., Murata, J., \& Kawabe, T. (2013). Model predictive control of vehicles on urban roads for improved fuel economy. IEEE Transactions on Control Systems Technology, 21, 831-841.

12. Kiam Heong, A., Chong, G., \& Li, Y. (2005). PID control system analysis, design, and technology. IEEE Transactions on Control Systems Technology, 13, 559-576. https://doi.org/10.1109/TCST.2005.847331.

13. Ko, Y., Song, B., \& Oh, Y. (2019). Mathematical analysis of environmental effects of forming a platoon of smart vehicles. Sustainability, 11, 571.

14. Kohut, N. J., Karl Hedrick, P. J., \& Borrelli, P. F. (2009). Integrating traffic data and model predictive control to improve fuel economy. IFAC Proceedings Volumes12th IFAC Symposium on Control in Transportation Systems, 42, 155-160

15. Larue, G. S., Malik, H., Rakotonirainy, A., \& Demmel, S. (2014). Fuel consumption and gas emissions of an automatic transmission vehicle following simple eco-driving instructions on urban roads. IET Intelligent Transport Systems, 8, 590-597.

16. Lei, C., van Eenennaam, E. M., Klein Wolterink, W., Ploeg, J., Karagiannis, G., \& Heijenk, G. (2012). Evaluation of CACC string stability using SUMO, Simulink, and OMNeT++. EURASIP Journal on Wireless Communications and Networking, 2012, 116.

17. Li, L., Wang, X., \& Song, J. (2017). Fuel consumption optimization for smart hybrid electric vehicle during a car-following process. Mechanical Systems and Signal ProcessingSignal Processing and Control challenges for Smart Vehicles, 87, 17-29.

18. Li, S., Li, K., Rajamani, R., \& Wang, J. (2011). Model predictive multi-objective vehicular adaptive cruise control. IEEE Transactions on Control Systems Technology, 19, 556-566.

19. Li, S., Li, K., Wang, J., Zhang, L., Lian, X., Ukawa, H., \& Bai, D. (2008). MPC based vehicular following control considering both fuel economy and tracking capability. In 2008 IEEE vehicle power and propulsion conference. Presented at the 2008 IEEE Vehicle Power and Propulsion Conference (pp. 1-6).

20. Luo, Y., Chen, T., Zhang, S., \& Li, K. (2015). Intelligent hybrid electric vehicle ACC with coordinated control of tracking ability, fuel economy, and ride comfort. IEEE Transactions on Intelligent Transportation Systems, 16, 2303-2308.

21. Ma, G., Ghasemi, M., \& Song, X. (2018). Integrated powertrain energy management and vehicle coordination for multiple connected hybrid electric vehicles. IEEE Transactions on Vehicular Technology, 67, 2893-2899.

22. Makridis, M., Fontaras, G., Ciuffo, B., \& Mattas, K. (2019). MFC free-flow model: Introducing vehicle dynamics in microsimulation. Transportation Research Record. https://doi.org/10.1177/0361198119838515.

23. Makridis, M., Mattas, K., Borio, D., Giuliani, R., \& Ciuffo, B. (2018). Estimating reaction time in adaptive cruise control system. In 2018 IEEE intelligent vehicles symposium (IV) (pp. 1312-1317).

24. Makridis, M., Mattas, K., \& Ciuffo, B. (2019). Response time and time headway of an adaptive cruise control. An empirical characterization and potential impacts on road capacity. IEEE Transactions on Intelligent Transportation Systems, 1-10. Early Access.

25. Mamouei, M., Kaparias, I., \& Halikias, G. (2018). A framework for user- and system-oriented optimisation of fuel efficiency and traffic flow in adaptive cruise control. Transportation Research Part C: Emerging Technologies, 92, 27-41.

26. Markschläger, P., Wahl, H.-G., Weberbauer, F., \& Lederer, M. (2012). Assistance system for higher fuel efficiency. Auto Tech Review, 1, 40-45.

27. MathWorks. (2019). 2D correlation coefficient https://uk.mathworks.com/help/ images/ref/corr2.html.

28. Munyaneza, O., Munyazikwiye, B. B., \& Karimi, H. R. (2015). Speed control design for a vehicle system using fuzzy logic and PID controller. In Presented at the 2015 international conference on fuzzy theory and its applications (iFUZZY) (pp. 56-61)

29. Osman, K., Rahmat, M. F., \& Ahmad, M. A. (2009). Modelling and controller design for a cruise control system (pp. 254-258). Presented at the 2009 5th International Colloquium on Signal Processing Its Applications. Penang.

30. Park, S., Rakha, H., Ahn, K., \& Moran, K. (2013). Fuel economy impacts of manual, conventional cruise control, and predictive eco-cruise control driving. International Journal of Transportation Science and Technology, 2, 227-242.

31. Raposo, A. (2017). The r-evolution of driving: From connected vehicles to coordinated automated road transport (C-ART). European Commission. Publications office of the European Union.

32. Themann, P., Bock, J., \& Eckstein, L. (2015). Optimisation of energy efficiency based on average driving behaviour and driver's preferences for automated driving. IET Intelligent Transport Systems, 9, 50-58.

33. Vajedi, M., \& Azad, N. L. (2016). Ecological adaptive cruise controller for plug in hybrid electric vehicles using nonlinear model predictive control. IEEE Transactions on Intelligent Transportation Systems, 17, 113-122. 
34. Wu, C., Zhao, G., \& Ou, B. (2011). A fuel economy optimization system with applications in vehicles with human drivers and autonomous vehicles. Transportation Research Part D: Transport and Environment, 16, 515-524.

35. Xie, S., Hu, X., Liu, T., Qi, S., Lang, K., \& Li, H. (2019). Predictive vehiclefollowing power management for plug-in hybrid electric vehicles. Energy, $166,701-714$.

36. Zhao, R. C., Wong, P. K., Xie, Z. C., \& Zhao, J. (2017). Real-time weighted multi-objective model predictive controller for adaptive cruise control systems. International Journal of Automotive Technology, 18, 279-292.

37. Zhao, W., Ngoduy, D., Shepherd, S., Liu, R., \& Papageorgiou, M. (2018). A platoon based cooperative eco-driving model for mixed automated and human-driven vehicles at a signalised intersection. Transportation Research Part C: Emerging Technologies, 95, 802-821.

38. Zhou, M., Jin, H., \& Wang, W. (2016). A review of vehicle fuel consumption models to evaluate eco-driving and eco-routing. Transportation Research Part D: Transport and Environment, 49, 203-218.

\section{Publisher's Note}

Springer Nature remains neutral with regard to jurisdictional claims in published maps and institutional affiliations.

\section{Submit your manuscript to a SpringerOpen ${ }^{\circ}$ journal and benefit from:}

- Convenient online submission

- Rigorous peer review

- Open access: articles freely available online

High visibility within the field

- Retaining the copyright to your article

Submit your next manuscript at $\boldsymbol{\nabla}$ springeropen.com 